\title{
Differential expression of tetraspanin CD9 in basal cell and squamous cell carcinomas of the skin and actinic keratosis
}

\author{
THOMAS ACH ${ }^{1,2}$, MIRJANA ZIEMER ${ }^{3}$, JENS DAWCZYNSKI ${ }^{2}$, \\ JÜRGEN STROBEL ${ }^{2}$, GEORG SAUER ${ }^{4}$ and HELMUT DEISSLER ${ }^{4}$ \\ ${ }^{1}$ Department of Ophthalmology, University Hospital Heidelberg, Heidelberg; \\ Departments of ${ }^{2}$ Ophthalmology and ${ }^{3}$ Dermatology, University Hospital Jena, Jena; \\ ${ }^{4}$ Department of Gynaecology and Obstetrics, University of Ulm, Ulm, Germany
}

Received May 22, 2009; Accepted September 24, 2009

DOI: 10.3892/ol_00000006

\begin{abstract}
Tetraspanins are potentially useful molecular markers that differentiate between tumour classes and subtypes, since members of this protein family were often found to be altered during malignant conversion and tumour progression. In this study, we analysed expression of the tetraspanin CD9 in the frequent cutaneous neoplasms basal cell carcinoma (BCC), squamous cell carcinoma (SCC) and actinic keratosis (AK), which is considered a precursor lesion (carcinoma in situ) from which an invasive SCC can develop. A moderate to strong CD9-specific staining of the tumour cells' plasma membranes was uniquely observed in all BCCs, SCCs and AKs. All SCCs showed additional intracellular CD9 which was rarely (20\%) seen in AKs. Semi-quantitative assessment of CD9 present in the plasma membranes of tumour cells of BCCs (mean staining intensity 1.91) and SCCs (3.64) reflected the different CD9 expression of normal precursor cells from which these tumours most likely originate. Although considered an intermediate stage in the development of SCCs, AKs did not show intense staining of the plasma membranes typical of normal keratinocytes or invasive SCCs $(\mathrm{p}=0.011)$ but only moderate intensity (mean 1.63). In BCCs, significantly $(\mathrm{p}=0.0005, \mathrm{n}=56)$ stronger CD9-specific immunoreactivity was seen in the inner regions of the tumours than at their sites of expansion. In summary, our results point to an important role of CD9 at the front of tumour expansion in BCCs and SCCs, and in the pathogenesis of invasive SCCs.
\end{abstract}

Correspondence to: Dr Helmut Deissler, Department of Obstetrics and Gynaecology, University of Ulm, Frauensteige 14, D-89075 Ulm, Germany

E-mail: helmut.deissler@uniklinik-ulm.de

Key words: tetraspanin, cutanous neoplasm, solar keratosis, immunohistochemistry

\section{Introduction}

The most frequent cutaneous neoplasms in aging Caucasian populations are basal cell carcinoma (BCC), squamous cell carcinoma (SCC) and actinic keratosis (AK), which is considered a carcinoma in situ (CIS) from which an invasive SCC can develop (1-4). Genesis of all these types of skin tumours, originating from epidermal keratinocytes or pluripotent basaloid cells, is initiated and driven by exposure to ultraviolet light. Accordingly, they are predominantly observed on sunexposed skin including forehead, nose, upper lip and lids. Although only few cases of metastasizing BCCs have been reported, clinically significant morbidity can be caused by deep and extensive destructive invasion of the surrounding tissue. In contrast, approximately $5 \%$ of invasive SCCs of the skin can form metastases by entering lymphatic or haematogenous vessels $(5,6)$. Therefore, surgical excision of these tumours is a curative treatment only during their early stages $(7,8)$. Accurate diagnosis of these tumours and their subclassification requires, in addition to macroscopic examination, careful histopathological assessment of the excised specimens. However, even those currently used methods are not perfectly reliable (9) and accuracy can be improved by identification and validation of additional differential molecular characteristics.

Tetraspanins are potentially useful molecular markers, since members of this protein family of transmembrane proteins were often found to be altered during malignant conversion and tumour progression, in accordance with their roles in a number of fundamental cellular processes, including adhesion, migration and intracellular signalling $(10,11)$. Different expression of a tetraspanin by histologically defined subtypes has recently been shown for ovarian carcinomas (12).

Correlations between expression of $\mathrm{CD} 9$, the best-studied tetraspanin, and clinical observations or relevant characteristics of tumour tissues and their cells were reported for many types of human cancer including melanoma (13). Other types of skin tumours have not been investigated with the exception of 5 analyzed cases of BCC (7).

In this initial study, we analysed CD9 expression of 80 epithelial neoplasms to reveal potential differences between 
Table I. Expression of CD9 by different classes and subtypes of non-melanoma skin tumours.

\begin{tabular}{lccccc}
\hline & & \multicolumn{2}{c}{ No. (CD9-positive) } & & \multicolumn{2}{c}{ Staining intensity } \\
\cline { 3 - 4 } Tumour type & No. & Membrane & Intracellular & & Periphery \\
\hline All & 80 & 78 & 57 & \\
BCCs & 56 & 54 & 41 & $1.91 \pm 0.75$ & $1.51 \pm 0.76$ \\
Superficial & 17 & 17 & 13 & $1.88 \pm 0.78$ & $1.41 \pm 0.94$ \\
Nodular & 21 & 19 & 11 & $1.71 \pm 0.78$ & $1.19 \pm 0.51$ \\
Sclerosing & 18 & 18 & 17 & $2.17 \pm 0.62$ & $2.00 \pm 0.59$ \\
SCCs & 14 & 14 & 14 & $3.64 \pm 0.50$ & $3.43 \pm 0.65$ \\
Invasive & 8 & 8 & 8 & $3.75 \pm 0.46$ & $3.63 \pm 0.52$ \\
Deeply invasive & 6 & 6 & 6 & $3.50 \pm 0.50$ & $3.16 \pm 0.69$ \\
AK & 10 & 10 & 2 & $1.63 \pm 0.52$ \\
\hline
\end{tabular}

To the observed intensities of CD9-specific staining, a score in the range 0-4 was assigned. For the different classes (BCC, basal cell carcinoma; SCC, squamous cell carcinoma; AK, actinic keratosis) and their subtypes, means of these scores and standard deviations are shown. CD9 staining of plasma membranes was assessed in the core of the tumour and at the borders of tumour expansion (periphery).

subtypes of carcinomas and between SCCs and their precursor AK lesions.

\section{Materials and methods}

Patients and tumour samples. Tissue samples collected from 80 patients of the Departments of Ophthalmology and Dermatology of the University Hospital in Jena after surgical excision of non-melanoma skin tumours. The tumours were removed from periocular locations or lids $(26 ; 32.5 \%)$, other parts of the head $(36 ; 45 \%)$ or other locations on the body (18; $22.5 \%$ ). Histopathological assessment of serial haematoxylin and eosin-stained sections, performed at the Department of Dermatology by one examiner (M.Z.), provided the basis for classification of the tumours in superficial, nodular or sclerosing BCCs, invasive SCCs with the distinct subclass of deeply invasive tumours and AK-type CIS lesions (Table I). Vertical tumour extension of $>4 \mathrm{~mm}$ defined deep invasion in the sub-classification of invasive SCCs in this study. Mean age of all patients was 72 years. Patients with BCCs were younger (mean age 69 years) than patients harbouring SCCs (81 years) or AKs (78 years).

Immunohistochemical staining. Immmunohistochemical staining of sections $(4 \mu \mathrm{m})$ of paraffin-embedded tissues was performed as previously described using a system with signal amplification through a multivalent link antibody (12). The CD9-specific primary antibody from NovoCastra (Newcastle upon Tyne, UK) was diluted 1:40 in Tris-buffered saline and allowed to bind overnight at $4^{\circ} \mathrm{C}$. Of all stained sections, parallel sections were processed with a similar amount of an isotypematched (mouse $\operatorname{IgG}_{1}$ from Southern Biotech, Birmingham, USA) control antibody to exclude non-specific binding.

CD9-specifc staining was assessed after counterstaining with hematoxylin by light microscopy and a score was assigned according to a linear scale from 0 (no staining) to
4 for the highest observed intensities. For each slide, scores indicating observed CD9-specific staining were recorded for several microscopic fields from the centre of the tumour mass and from the tumours' peripheries. In addition, both intracellular and cell surface staining of CD9 were analysed.

Data analysis. For the histopathologically defined classes and subtypes of tumours, means of intensity scores and standard deviations were calculated. To reveal potentially significant $(\mathrm{p}<0.05)$ differences between pairs of groups, the two-sided Mann-Whitney test was used, and for paired groups of variables from the same tissue sample (staining at the tumour core vs. periphery) the Wilcoxon test. Both were included in the SPSS Statistics (version 16, SPSS, Chicago, IL, USA) software package.

\section{Results}

CD9 expression of the main types of non-melanoma skin tumour cells was determined by immunohistochemical staining of tissue sections. A moderate to strong CD9-specific staining of the tumour cells' plasma membranes was uniquely observed in all BCCs, SCCs and AK-type carcinomas in situ (Fig. 1). An additional granular intracellular staining was significantly different between the investigated types of tumours. All invasive SCCs showed intracellular CD9, whereas this subcellular location was rarely $(20 \%)$ seen in AKs. This difference was calculated with Fisher's exact test to be highly $(\mathrm{p}=0.001)$ significant. Intracellular staining was also observed in sclerosing BCCs, but only in a fraction of superficial and nodal BCCs (Table I).

Semi-quantitative assessment of CD9 present in the plasma membranes of tumour cells of BCCs (mean staining intensity 1.91) and invasive SCCs (3.64) reflected the different CD9 expression of normal undifferentiated basaloid cells $(\sim 2.5)$ and keratinocytes $(\sim 3.5)$ from which these tumours most likely 

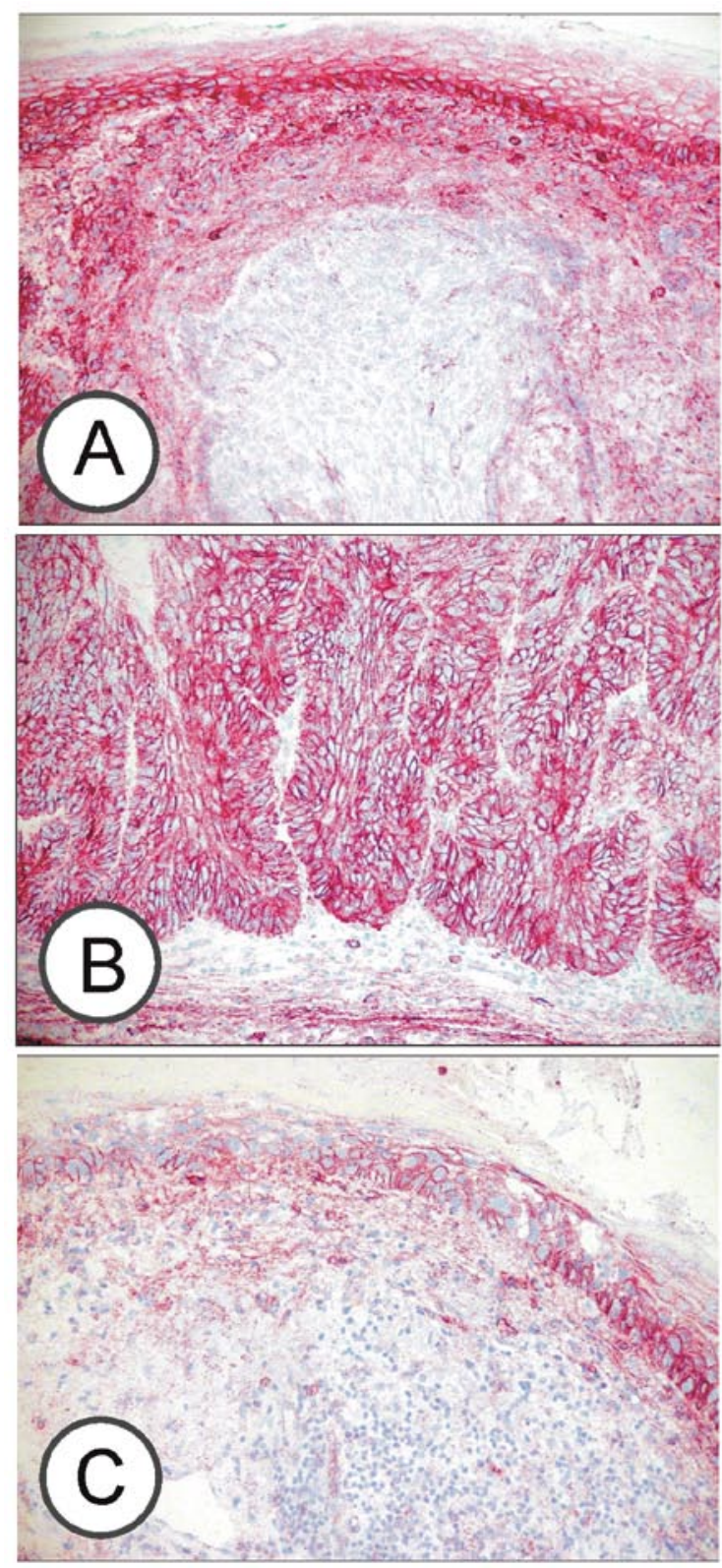

Figure 1. CD9-specific staining of BCCs, invasive SCCs and AKs. Immunohistochemical CD9-specific stainings of representative cases of the main types of non-melanoma skin tumours are shown. Immunoreactivity is indicated by red staining, counterstaining with hematoxylin resulted in blue nuclei. (A) Nodular BCC with weak staining of cells in the core of the tumour compared with stronger expression of CD9 by tumour cells at the periphery of a tumour nodule. Intact epidermis shows the typical strong staining of CD9, which is also observed in the stroma. (B) Deeply invading SCC with very strong expression of CD9 by all tumour cells. (C) AK-type carcinoma in situ with only weak to moderate staining of CD9 compared to invasive SCCs (B) and adjacent normal epidermis of which a part is shown at the right edge.

originate. Surprisingly, investigated AKs did not show intense staining of the plasma membranes typical of normal keratinocytes or invasive SCCs ( $\mathrm{p}=0.011$; Mann-Whitney test) but only moderate (mean 1.63) intensity (Figs. 1 and 2A). Within each group, membrane stainings of subtypes of BCCs and invasive SCCs were not significantly different.

Since CD9 is a tetraspanin involved in cell adhesion and migration, CD9-specific immunoreactivity was assessed both in the cores of BCCs and invasive SCCs, and at their
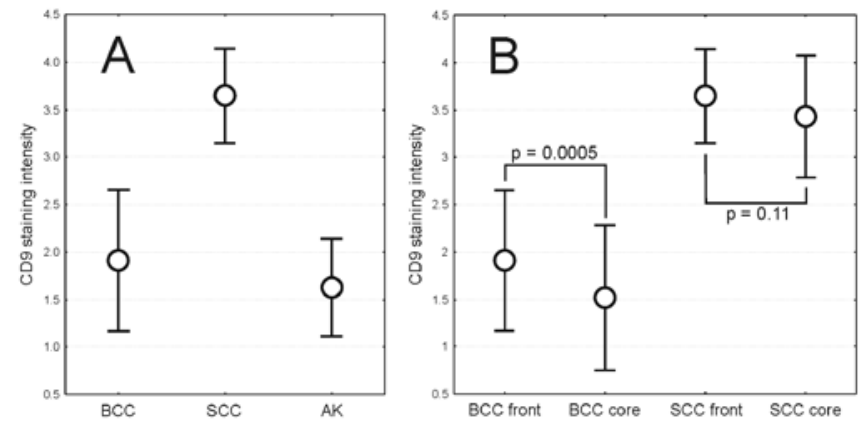

Figure 2. Comparison of CD9 staining intensities determined for BCCs, invasive SCCs and AKs and for different regions of the tumour tissues. (A) CD9-specific staining of plasma membranes was stronger in invasive SCCs than in BCCs. Notably, keratinocytes forming AK-type carcinomas in situ expressed CD9 substantially weaker than SCC cells. (B) CD9 expression was pronounced at the borders of tumour expansion (front) compared to the central parts of the tumours (core). For BCCs, the difference was calculated to be significant with the Wilcoxon test. The mean values of staining intensities (circles) with corresponding standard deviations (whiskers) are shown.

advancing borders. Stronger staining was clearly observed at the peripheries of BCCs ( $\mathrm{p}=0.0005, \mathrm{n}=56$; Wilcoxon test), for the fewer cases of invasive SCCs this difference did not yet reach statistical significance ( $\mathrm{p}=0.11$; Fig. $2 \mathrm{~B})$.

\section{Discussion}

We analyzed expression of the tetraspanin CD9 by cells forming BCCs, SCCs or AKs which are considered carcinomas in situ from which invasive SCCs can develop $(3,4)$. Despite the limited number of cases, significant differences were observed. In SCC and BCC cells, strong CD9 expression of the normal cells from which these originate, i.e. keratinocytes and basaloid precursor cells, appeared to be conserved. However, the particularly strong CD9 expression of normal keratinocytes was found to be strongly decreased in AK lesions, which suggests its down-regulation at the $\mathrm{AK}$ stage of carcinogenesis followed by complete restoration during transition to invasive SCC. One could speculate that lower amounts of CD9 promote non-invasive expansion of AK cells through decreased attachment to the extracellular matrix (ECM) because of the CD9 interaction with integrins in keratinocytes (14-16) which might also affect proliferation. At the stage of invasive SCC, up-regulation of CD9 could contribute to processes that allow invasion through ECM barriers in the tissue which was observed in advanced stages of cervical carcinomas (17). However, since a low expression of CD9 was frequently identified to be an indicator of poor prognosis of patients and further progression in a number of tumour types, including melanoma (13) and SCCs of other locations (18-21), a subsequent study should focus on potential differences of tetraspanin expression in metastatic cutaneous SCCs. The observed moderate expression of CD9 in the plasma membranes of BCC cells reflect amounts of this tetraspanin found in normal basal cells and, therefore, appeared to be unaltered in the carcinoma cells. Notably, significantly more CD9 was expressed at advancing borders of the expanding tumours than in their inner regions. This is in accordance with CD9's role in promoting cell migration, which was demonstrated for various malignant and 
non-malignant cell types, e.g. microvascular endothelial cells (22). Since migration is a complex process involving both local detachment and attachment of cells, increased expression of a tetraspanin such as CD9 can promote or inhibit movement of cells, depending strongly on the cell and tissue type and environmental factors. In BCCs, increased amounts of CD9 at sites of expansion suggest a stimulation of migration. In AK cells, stimulation of migration might be achieved by downregulation of $\mathrm{CD} 9$, before tumour progression gives rise to invasive SCC cells in which the pro-migratory effect of CD9 dominates again.

In this study we included the most frequent classes and subtypes of non-melanoma skin tumours with a malignant potential. Although AKs, invasive SCCs and BCCs differed in their expression of $\mathrm{CD} 9$, there was no indication of subtypespecific expression among BCC and SCC subtypes which would have been helpful in their histopathological assessment. For BCCs and SCCs, our results point to an important role of CD9 at the front of tumour expansion. The differential expression of CD9 by AK-type carcinomas in situ and invasive SCCs suggests that it is switched off and on during the development of a SCC. This observation provides the basis for further investigation of the roles of tetraspanins in the pathogenesis of SCCs.

\section{Acknowledgements}

We thank D. Lamm and S. Feldrappe (Department of Dermatology, University of Jena) and J. Windisch (Department of Gynaecology and Obstetrics, University of Ulm) for their expert technical assistance.

\section{References}

1. Diepgen TL and Mahler V: The epidemiology of skin cancer. Br J Dermatol 146: 1-6, 2002.

2. Leiter $U$ and Garbe C: Epidemiology of melanoma and nonmelanoma skin cancer - the role of sunlight. Adv Exp Med Biol 624: 89-103, 2008

3. Frost CA and Green AC: Epidemiology of solar keratoses. Br J Dermatol 131: 455-464, 1994.

4. Ortonne JP: From actinic keratosis to squamous cell carcinoma. Br J Dermatol 146: 20-23, 2002.

5. Kwa RE, Campana K and Moy RL: Biology of cutaneous squamous cell carcinoma. J Am Acad Dermatol 26: 1-26, 1992.

6. Chin CW, Foss AJ, Stevens A and Lowe J: Differences in the vascular patterns of basal and squamous cell skin carcinomas explain their differences in clinical behaviour. J Pathol 200: 308-313, 2003.
7. Anthony ML: Surgical treatment of nonmelanoma skin cancer. AORN J 71: 552-558, 560, 2000.

8. Neville JA, Welch E and Leffell DJ: Management of nonmelanoma skin cancer in 2007. Nat Clin Pract Oncol 4: 462-469, 2007.

9. Jagdeo J, Weinstock MA, Piepkorn M and Bingham SF: Reliability of the histopathologic diagnosis of keratinocyte carcinomas. J Am Acad Dermatol 57: 279-284, 2007.

10. Hemler ME: Tetraspanin functions and associated microdomains. Nat Rev Mol Cell Biol 6: 801-811, 2005.

11. Hemler ME: Targeting of tetraspanin proteins - potential benefits and strategies. Nat Rev Drug Discov 7: 747-758, 2008.

12. Scholz CJ, Kurzeder C, Koretz K, Windisch J, Kreienberg R, Sauer G and Deissler H: Tspan-1 is a tetraspanin preferentially expressed by mucinous and endometrioid subtypes of human ovarian carcinomas. Cancer Lett 275: 198-203, 2009.

13. Si Z and Hersey P: Expression of the neuroglandular antigen and analogues in melanoma. CD9 expression appears inversely related to metastatic potential of melanoma. Int $\mathrm{J}$ Cancer 54: 37-43, 1993.

14. Baudoux B, Castanares-Zapatero D, Leclercq-Smekens M, Berna N and Poumay Y: The tetraspanin CD9 associates with the integrin alpha6beta4 in cultured human epidermal keratinocytes and is involved in cell motility. Eur J Cell Biol 79: 41-51, 2000.

15. Jones PH, Bishop LA and Watt FM: Functional significance of CD9 association with beta 1 integrins in human epidermal keratinocytes. Cell Adhes Commun 4:297-305, 1996.

16. Okochi H, Kato M, Nashiro K, Yoshie O, Miyazono K and Furue M: Expression of tetra-spans transmembrane family (CD9, CD37, CD53, CD63, CD81 and CD82) in normal and neoplastic human keratinocytes: an association of CD9 with alpha 3 beta 1 integrin. Br J Dermatol 137: 856-863, 1997.

17. Sauer G, Windisch J, Kurzeder C, Heilmann V, Kreienberg R and Deissler H: Progression of cervical carcinomas is associated with down-regulation of CD9 but strong local re-expression at sites of transendothelial invasion. Clin Cancer Res 9: 6426-6431, 2003.

18. Erovic BM, Pammer J, Hollemann D, Woegerbauer M, Geleff S, Fischer MB, Burian M, Frommlet F and Neuchrist C: Motilityrelated protein-1/CD9 expression in head and neck squamous cell carcinoma. Head Neck 25: 848-857, 2003.

19. Uchida S, Shimada Y, Watanabe G, Li ZG, Hong T, Miyake M and Imamura M: Motility-related protein (MRP-1/CD9) and KAI1/CD82 expression inversely correlate with lymph node metastasis in oesophageal squamous cell carcinoma. Br J Cancer 79: 1168-1173, 1999.

20. Kusukawa J, Ryu F, Kameyama T and Mekada E: Reduced expression of CD9 in oral squamous cell carcinoma: CD9 expression inversely related to high prevalence of lymph node metastasis. J Oral Pathol Med 30: 73-79, 2001.

21. Mhawech P, Dulguerov P, Tschanz E, Verdan C, Ares C and Allal AS: Motility-related protein-1 (MRP-1/CD9) expression can predict disease-free survival in patients with squamous cell carcinoma of the head and neck. Br J Cancer 90: 471-475, 2004.

22. Deissler H, Kuhn EM, Lang GE and Deissler H: Tetraspanin CD9 is involved in the migration of retinal microvascular endothelial cells. Int J Mol Med 20: 643-652, 2007. 